\title{
Gerenciamento dos Resíduos Provenientes do Setor de Tecnologia da Informação do Instituto Federal Fluminense
}

\author{
Tiago Gomes Da Silva Ribeiro - (UCAM e IF Fluminense) - tribeiro@iff.edu.br \\ Acacio Romeu Rodrigues Santos - (UCAM) - romeu.rodrigues@manabi.com \\ Milton Erthal Júnior - (UCAM e IF Fluminense) - miltonerthal@hotmail.com
}

Resumo: Os resíduos eletrônicos já representam 5\% de todo o lixo produzido pela humanidade. Neste artigo foi realizado um estudo de caso para identificar qual o melhor fluxo para o descarte dos resíduos sólidos gerados pelo setor de tecnologia da informação do Instituto Federal Fluminense - IFF. Na pesquisa, foi realizada uma coleta de dados através de entrevista qualificada aplicada aos integrantes do setor de tecnologia da informação e almoxarifado do IFF. Com o fluxo proposto neste trabalho, ficará claro o caminho a ser seguido pelo e-lixo, evitando que ele fique parado ocupando espaços preciosos enquanto aguarda definição de seu destino. Outro benefício é a possibilidade de reaproveitamento de componentes ou elementos básicos do lixo eletrônico, seja em outras instituições sem fins lucrativos, seja gerando ocupação e renda para catadores e recicladores, possibilidade muito positiva em um país em desenvolvimento como o Brasil. Enfim, será mais fácil dar uma destinação final aos resíduos sólidos gerados pelo IFF, contribuindo para a preservação do meio ambiente a redução do volume de resíduos sólidos.

Palavras-chave: Resíduos Sólidos; e-lixo; Lixo Eletrônico.

Abstract: Nowadays, the electronic waste (e-waste) is five \% of all the trash produced by men's. The aim of this article was investigate the beast way to discharge the electronic waste produced by the department of technology information of Instituto Federal Fluminense (IFF). A case study was conducted in the warehouse of information technology of IFF and by literature review. The flow of e-waste starts with the users and arrives in technology department after the detection of the equipment problem. The machine is examined to define the end of e-waste: reuse, donation or discharge. In this way we can avoid take up unnecessary space in IFF. This study open opportunities to reuse the e-waste by others educations institutes, taking incomes and occupations to scavengers and encourage the recycling industry. This work will allow the employers of IFF how to procedure with the e-waste and vacate the section of information technology. This kind of initiative contributes with the reduction of solid waste production and with environmental preservation.

\section{Key words: waste, e-waste, information technology.}

\section{Introdução}

Resíduos são todas as “coisas” indesejadas geradas na produção ou consumo de bens. A massa de resíduos sólidos gerada pela sociedade industrial é muito superior à massa de produtos consumidos: todos os bens que consumimos ao final da sua vida útil serão resíduos; todo e qualquer processo de mineração, extração ou industrial gera resíduos (RIBEIRO \& MORELLI, 2009). 
Nos nossos dias, existem parcerias que enfrentam os sérios desafios para o lixo se tornar cada vez algo melhor, mas seu objetivo é a tentativa de reduzir os danos socioambientais provocados pela destinação inadequada dos resíduos sólidos, tendo em vista promover benefícios econômicos, sociais e ambientais para a sociedade, incluindo a valorização do material reciclável, a geração de empregos e fonte de renda estável para os catadores de lixo, a inclusão social destas pessoas e a redução do volume e dos impactos ambientais do lixo (PIRANI, 2010).

Os resíduos eletrônicos já representam 5\% de todo o lixo produzido pela humanidade. O Brasil produz 2,6Kg de lixo eletrônico por habitante, o equivalente a menos de $1 \%$ da produção mundial de resíduos do mundo, porém a indústria eletrônica continua em expansão (FERRREIRA et. al 2010).

Lixo eletrônico é o nome dado aos resíduos resultantes da rápida obsolescência de equipamentos eletrônicos que inclui computadores, celulares, geladeiras, televisores, impressoras e outros dispositivos (OLIVEIRA et. al 2009).

Considerando que o mundo hoje vive apenas o principio da revolução tecnológica, onde tudo e todos serão geridos pelos recursos advindos da tecnologia da informação, a preocupação com este tema, TI, torna-se imprescindível, pois fica explicito, mais uma vez, a importância de se estabelecer maior harmonia entre os sistemas antropocêntricos e meio ambiente (PEREIRA, 1993)

A falta de espaço adequado para guardar os resíduos é um problema grave para o Instituto Federal Fluminense (IFF), e a cada dia mais e mais equipamentos vão ficando obsoletos.

Neste sentido este trabalho tem por objetivo propor um fluxo de gerenciamento dos resíduos provenientes do setor de TI do IFF.

\section{Referencial Teórico}

A urbanização vem crescendo de forma acelerada. Em 1800, apenas cinco em cada cem habitantes do mundo moravam em cidades. Desde então, este número aumentou para 40. O homem está saindo da zona rural e indo para a cidade. Nos nossos dias, com a maioria das pessoas vivendo nas cidades e com o avanço mundial da indústria, provocando mudanças nos hábitos de consumo da população, vem-se gerando um lixo diferente em quantidade e diversidade.

Começando a olhar o problema de uma janela mais ampla, Doyle (2007) apresenta dados que indicam a crescente produção de REE - Resíduos Elétricos e Eletrônicos no mundo. Os REEs estão entre as categorias de lixo de mais alto crescimento e em breve devem atingir a marca dos 40 milhões de toneladas anuais, o suficiente para encher uma fileira de caminhões de lixo que se estenderia por metade do planeta.

Segundo SEI - Sustainable Eletronics Iniciative, da Universidade de Illinois, consultada em http://www.sustainelectronics.illinois.edu/policy/state.cfm, 03/04/2014, 06:27, a Califórnia é atualmente o único estado dos Estados Unidos da América com ARF Advanced Recycling Fee (Taxa Prévia de Reciclagem). A maioria dos estados tem leis de EPR - Extended Producer Responsability, que responsabilizam os fabricantes pela coleta e disposição dos produtos eletrônicos ao fim de sua vida (EOL - End-of-life).

Kahhat \& Williams (2012) indicam que, ainda nos EUA, 40 milhões de computadores usados (desktops, sem seus monitores, e laptops), em 2010, entraram no gerenciamento de fim de vida, dos quais 30\% foram reusados domesticamente, 6-29\% foram exportados, $17-21 \%$ foram para aterros e $20-47 \%$ foram coletados para reciclagem.

Segundo Chi et al (2011), na China, que produz, exporta e consome a maior porção de equipamentos eletroeletrônicos do mundo (Yang, 2008), o e-lixo, sendo uma das maiores 
fontes de metais pesados e poluentes orgânicos em lixos municipais e o fluxo de resíduos que cresce mais rápido, tem se tornado um sério problema, bem como em outros países asiáticos em desenvolvimento (Bertram et al., 2002). Não somente pela geração doméstica de tremenda quantidade de e-lixo devida ao rápido crescimento das taxas de consumo, mas também pelo recebimento de enormes quantidades de dispositivos e equipamentos de TI (Tecnologia da Informação) de países estrangeiros (Brigden et al., 2008).

Na China, e-lixo é largamente reciclado pelo setor informal, onde numerosos trabalhadores muito mal pagos aplicam métodos rudimentares e poluidores na separação de componentes reusáveis e na recuperação de metais contidos. Essas práticas se dão sob as mais primitivas circunstâncias, expondo trabalhadores a extensos riscos à saúde (Williams, 2005).

Segundo Ni \& Zeng (2009) a China tem importado e-lixo por mais de dez anos, recebendo entre 50 e $80 \%$ dos computadores obsoletos dos Estados Unidos. Percebe-se que a falta de leis e regras na China é a razão principal para a situação quase fora de controle hoje existente.

Para Sinha-Khetriwal et al (2005) são necessárias, primeiro, condições logísticas especiais para coletar lixo eletrônico. E como ele contém várias substâncias perigosas extremamente prejudiciais à saúde humana e ao meio ambiente, é necessário tratamento adequado para sua disposição, de modo a prevenir a fuga e dissipação de tóxicos no ambiente.

Ao mesmo tempo, ele é uma rica fonte de metais como ouro, prata e cobre, que podem ser recuperados de volta ao ciclo de produção. Essa característica tem feito sua reciclagem um negócio lucrativo tanto nos países desenvolvidos quanto nos emergentes.

Sinha-Khetriwal et al (2005) escolheram a Suíça e a Índia para uma comparação dos modelos de reciclagem de lixo eletrônico implementados nos dois países.

A Suiça foi escolhida por ter sido o primeiro país a implementar um sistema organizado para toda a indústria para coleta e reciclagem de lixo eletrônico.

A lei Suiça enfatiza o princípio poluidor-pagador e tem incentivado a redução, o reuso e a reciclagem de lixo. Um dos pilares do sistema é o financiamento segurado da coleta e reciclagem através de uma taxa prévia de reciclagem (Advanced Recycling Fee), embutida nos produtos e que pode ser recuperada na devolução. Controles independentes são facilmente aplicáveis, tornando transparente a manutenção da qualidade e dos padrões ambientais dos recicladores.

Ao contrário da Suíça, onde os consumidores pagam uma taxa de reciclagem, na Índia os coletores de lixo é que pagam a consumidores um preço por seus equipamentos. Os pequenos coletores em conjunto vendem a comerciantes que segregam e classificam os diferentes tipos de lixo e revendem aos recicladores. Aqui o principal incentivo a esses agentes é o lucro e não consciência ambiental ou social.

Conforme Rodrigues (2007) constata em seu estudo, existe uma lacuna no que diz respeito ao pós-consumo dos resíduos eletroeletrônicos no Brasil. Observou-se uma grande carência de empresas especializadas no gerenciamento desses resíduos, além de um desinteresse do mercado secundário de materiais para os resíduos eletrônicos (sucatas), tendo como consequência o descarte inadequado desses resíduos nos locais de destinação de resíduos domiciliares.

No Brasil, a geração do lixo ainda é, em sua maioria, de procedência orgânica; contudo, nos últimos anos vem se incorporando o modo de consumo de países ricos, o que tem levado a uma intensificação do uso de produtos descartáveis (CARVALHO, 2014). 


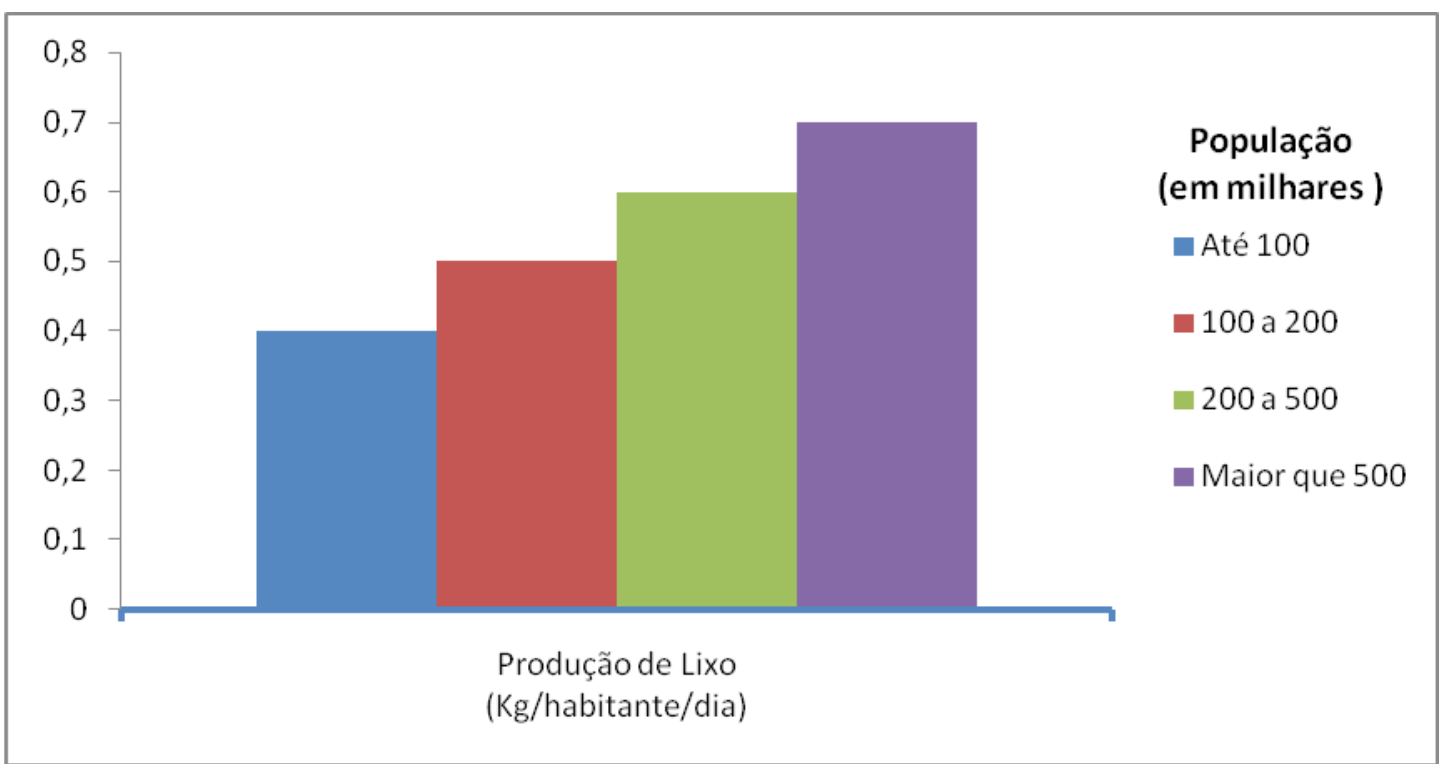

Figura 1 - Gráfico da produção de lixo em quilos por habitante por dia no Brasil.

Na figura 1 foram considerados os resíduos de origem domiciliar, ou seja, não foram contabilizados os resíduos gerados em indústrias, em podas, na limpeza de vias públicas e de córregos e outros. Nota-se que quanto mais a população aumenta em milhares de habitantes, aumenta também a produção de resíduos gerados pelos mesmos (TENÓRIO e ESPINOSA, 2004).

Para PIRANI (2010) a definição de resíduos sólidos e lixo tem uma distinção nos sentidos, sendo estes então diferentes, mas parecidos. O termo resíduo sólido está ligado à crescente especialização do discurso em relação ao lixo e também a uma mudança sociocultural e econômica na forma de encarar os rejeitos. O lixo por sua vez é entendido como algo velho e inútel, que já não se usa e se joga fora. Com a substituição da palavra lixo por resíduos sólidos, há uma alteração na relação das pessoas com o material descartado, que passa a ser visto como algo possível de ser reaproveitado e de gerar lucros financeiros e ambientais.

Existem várias formas para se classificarem os resíduos sólidos; os meios mais comuns são quanto aos riscos potenciais de contaminação do meio ambiente. São classificados em: Classe I - Perigosos; Classe II - Não Perigosos. Os Não Perigosos são classificados como Não-inertes ou Inertes (ABNT, 2004).

- Classe I: São Aqueles que em função de suas características de inflamabilidade, corrosividade, reatividade, toxicidade ou patogenicidade, apresentam riscos à saúde ou ao meio ambiente (RIBEIRO \& MORELLI, 2009).

- Casse II Não Perigosos:

- Não inertes: São aqueles que podem apresentar características de combustibilidade, biodegradabilidade ou solubilidade.

- Inertes: São aqueles que, por suas características intrínsecas, não oferecem riscos à saúde e que não apresentam constituintes solúveis em água em concentrações superiores aos padrões de portabilidade (RIBEIRO \& MORELLI, 2009).

Os lixões são muitos diferentes dos aterros sanitários, estes levam em consideração critérios ecológicos ou sanitários. Calcula-se que $47 \%$ dos municípios destinam seus resíduos em aterros sanitários e $22 \%$ em aterros controlados. 30\% para lixões, 0,4\% para compostagem, e apenas $0,1 \%$ seguem para a triagem e $20 \%$ da população brasileira ainda não possuem o serviço de coleta seletiva e reciclagem (PIRANI, 2010). 


\section{Metodologia}

No levantamento das informações em relação às composições e ao volume dos resíduos sólidos gerados pelo setor de tecnologia da informação, foi realizada uma coleta de dados através de entrevista qualificada aplicada aos integrantes do setor de tecnologia da informação e almoxarifado do IFF, onde procurou-se identificar o melhor fluxo de descarte dos resíduos.

Os setores foram questionados em relação ao caminho percorrido pelo resíduo até o seu descarte final no IFF. Foi questionado também se o resíduo é separado do lixo ou e se existe algum lugar que separa o lixo do resíduo.

\section{Estudo de Caso}

Informações obtidas no Setor de Patrimônio do IFF apontam a existência nos seus bens patrimoniais exatos 10.553 equipamentos de informática entre impressoras, notebooks, monitores, computadores, tablets, no-breaks, estabilizadores, scanners, webcams, etc. A figura 2, 3 e 4 demostram alguns equipamentos obsoletos precisando ser descartados abrigados em um espaço aguardando a definição de seu destino.

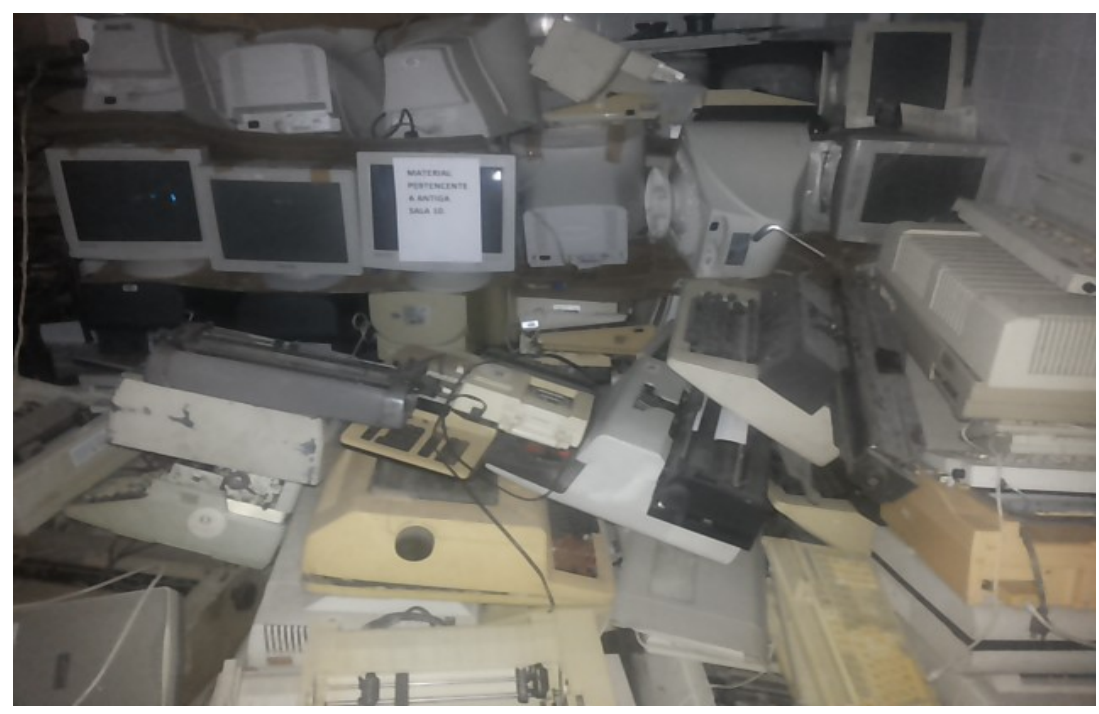

Figura 2 - Equipamentos obsoletos do Instituto Federal Fluminense

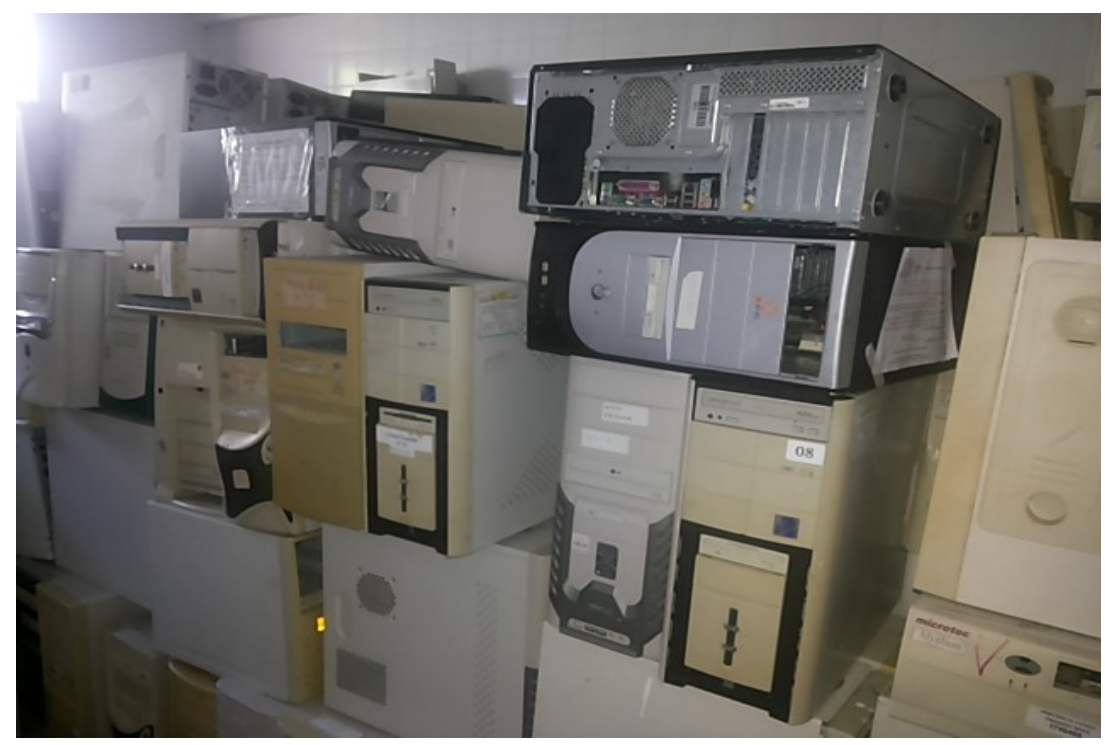


Figura 3 - Equipamentos obsoletos do Instituto Federal Fluminense

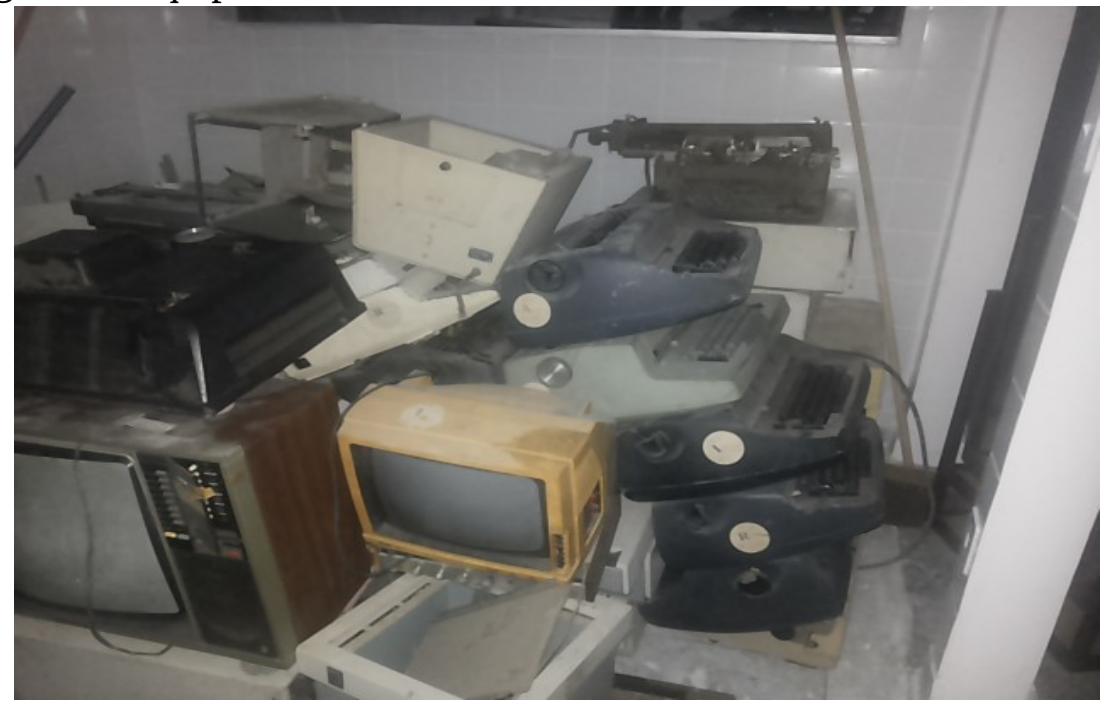

Figura 4 - Equipamentos obsoletos do Instituto Federal Fluminense

Os equipamentos das figuras acima possuem um grande teor de elementos de alto valor; na tabela 1 descreve-se a quantidade agregada presente em cada quilo de placa de circuito, de onde é possível coletar os seguintes miligramas:

\begin{tabular}{lccc}
\hline Elemento & Computador (placa-mãe) & Celular & Calculadora \\
\hline Ouro & 250 & 350 & 50 \\
Prata & 1.000 & 1.390 & 260 \\
Paládio & 110 & 210 & 5 \\
Cobre & 200.000 & 130.000 & 30.000 \\
Alumínio & 5.000 & 1.000 & 5.000 \\
\hline
\end{tabular}

Tabela 1: Teor médio de elementos de alto valor agregado presentes em placas de circuito impresso (mg kg-1). Fonte: Oliveira et al, 2010.

\section{Resultados}

A figura abaixo demonstra a proposta de como de como devem ser tratados os equipamentos de TI dentro da instituição desde seu uso até o descarte. 


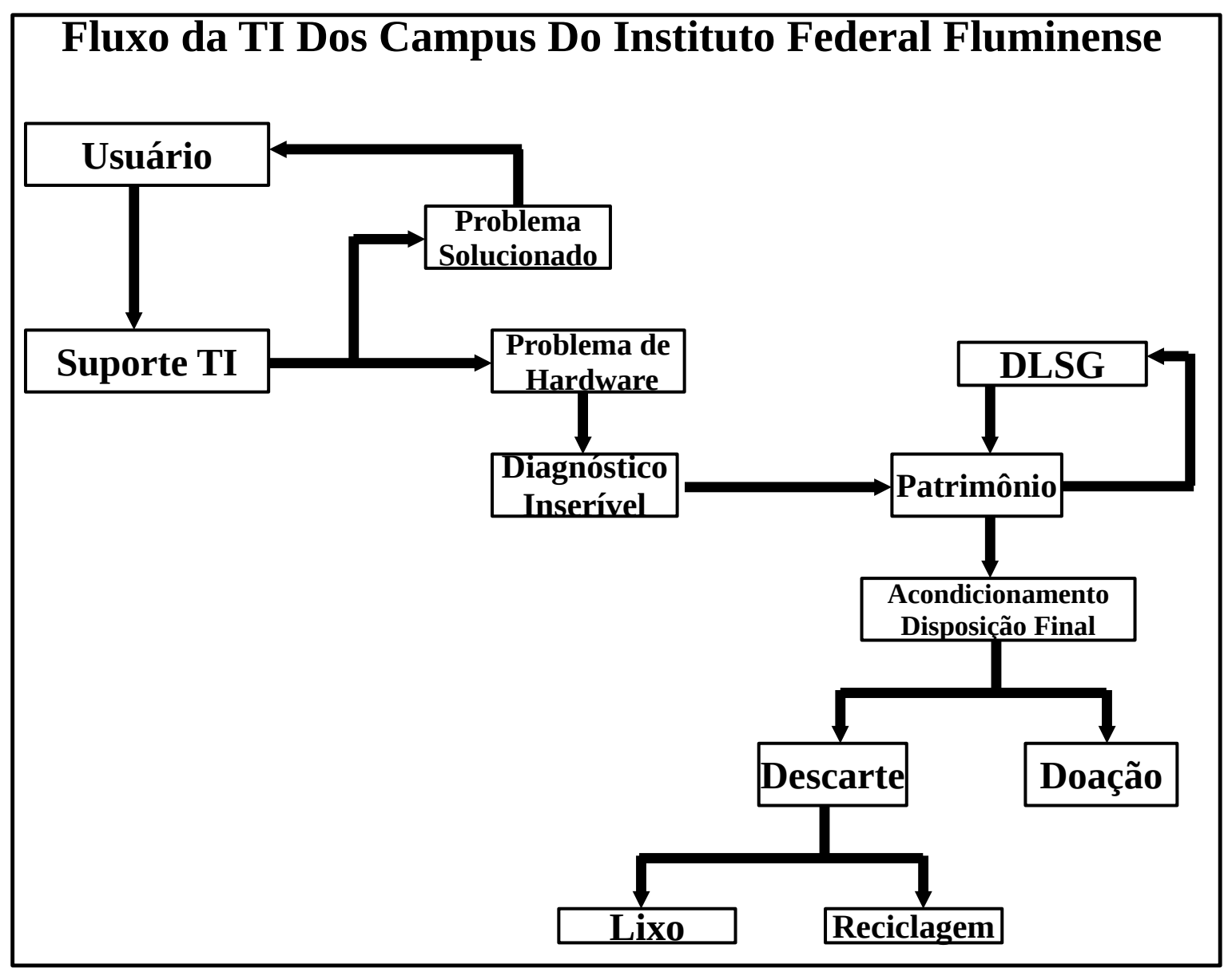

Figura 5 - Fluxo proposta para o descarte dos resíduos gerados pelo setor de tecnologia da informação

Legenda:

1 Usuário: usuários de equipamentos de tecnologia da informação.

2 Suporte TI: Equipe de Tecnologia da Informação do IFF.

3 Patrimônio: Setor de controle dos bens do IFF.

Problema Solucionado: Defeito reportado pelo usuário solucionado.

Problema de Hardware: Defeito físico nos equipamentos de Tecnologia da Informação.

DLSG: Departamento de Logística e Serviços gerais do Ministério do planejamento, orçamento e gestão.

Doação: Procede a doação para instituições sem fins lucrativos ou e/ou de interesse público.

Descarte: Procede a descarte para catadores em geral.

Disposição Final: Aguardando envio para doação ou descarte

Lixo: descarte direto ao lixo normal

Reciclagem: Encaminhar os resíduos a uma empresa especializada em reciclagem

Partindo do usuário todos os problemas passam pela equipe de suporte TI onde é diagnosticado se o problema é de hardware ou software. Os problemas de softwares são resolvidos e o equipamento retorna ao usuário. Já para os de hardware, é necessário efetuar o conserto ou a troca do mesmo. Se o custo da manutenção for maior que $50 \%$ do preço de mercado, o equipamento é considerado como sucata para retirada de peça para conserto de outros. O hardware com defeito é descartado, considerado inserível para o IFF.

Com o descarte, o hardware é encaminhado ao setor de patrimônio para efetuar a baixa do mesmo. Seguindo o DECRETO No 99.658/90, o setor de patrimônio relaciona todos os 
equipamentos inservíveis para o IFF e encaminha a lista para o DLSG (Departamento de Logística e Serviços Gerais do Ministério do Planejamento, Orçamento e Gestão). Esse departamento tem 30 dias para manifestar interesse pelos equipamentos e remanejá-los para outras instituições. Em caso de não manifestação pelo DLSG, os equipamentos ficam automaticamente disponíveis para doação ou descarte.

\section{Conclusão}

O Instituto Federal Fluminense, através da Diretoria de TI, concorda que a instituição está apenas no princípio, no que tange à implementação de políticas ambientalmente corretas como o conceito de TI Verde.

Diferentemente de instituições particulares que investem no meio ambiente objetivando o crescimento dos lucros, as instituições federais seguem exclusivamente a legislação vigente, as quais não demonstram preocupações mais profundas relacionadas ao meio ambiente.

Com o fluxo proposto aos setores de tecnologia da informação e almoxarifado, ficará claro o caminho a ser seguido pelo e-lixo, evitando que ele fique parado ocupando espaços preciosos enquanto aguarda definição de seu destino.

Outro benefício do fluxo proposto é a possibilidade de reaproveitamento de seus componentes ou elementos básicos, seja em outras instituições sem fins lucrativos, seja gerando ocupação e renda para catadores e recicladores, possibilidade muito positiva em um país em desenvolvimento como o Brasil.

Enfim, será mais fácil dar uma destinação final aos resíduos sólidos gerados pelo IFF, contribuindo para a preservação do meio ambiente a redução do volume de resíduos.

Contudo, para que instituições como o IFF mudem sua posição em relação aos problemas ambientais atualmente enfrentados, o primeiro passo é rever as leis pertinentes aos seus processos e procedimentos como é o caso do Plano de Lei Sobre Resíduos Sólidos que aponta que instituições no âmbito federal deverão passar a adquirir equipamentos reciclados ou recicláveis.

\section{Referencias Bibliográficas}

ASSOCIAÇÃO BRASILEIRA DE NORMAS TÉCNICAS. NBR 10.004: Resíduos sólidos Classificação. 2 ed. Rio de Janeiro: Impresso no Brasil, 2004.

CARVALHO, 2014, Lixo: conseqüências, desafios e soluções. Acesso em 25/02/14 página disponível no site WWW.conedcursos.com.br.

CHI, Xinwen et al. Informal electronic waste recycling: a sector review with special focus on China. Waste Management, v. 31, n. 4, p. 731-742, 2011.

FERREIRA, Dérick da Costa; SILVA, Josivan Bezerra da; GALDINO, Jean Carlos da Silva. Reciclagem do e-lixo (ou lixo eletro-eletrônico). Rio de Janeiro, nov. 2008.

KAHHAT, Ramzy; WILLIAMS, Eric. Materials flow analysis of e-waste: Domestic flows and exports of used computers from the United States. Resources, Conservation and Recycling, v. 67, p. 67-74, 2012.

MANOMAIVIBOOL, Panate. Extended producer responsibility in a non-OECD context: The management of waste electrical and electronic equipment in India. Resources, Conservation 
and Recycling, v. 53, n. 3, p. 136-144, 2009.

NI, Hong-Gang; ZENG, Eddy Y. Law enforcement and global collaboration are the keys to containing e-waste tsunami in China. Environmental science \& technology, v. 43, n. 11, p. 3991-3994, 2009.

OLIVEIRA, Rafael da Silva; GOMES, Elisa Silva; AFONSO, Júlio Carlos. O Lixo Eletroeletrônico: Uma Abordagem para o Ensino Fundamental e Médio. O Lixo Eletroeletrônico, Belo Horizonte, v. 32, n. 4, p.240-247, 05 out. 2010. Mensal.

PEREIRA NETO, João Tinôco. Minimização e aproveitamento de resíduos sólidos. In: SIMPÓSIO NACIONAL DE GERENCIAMENTO AMBIENTAL NA INDÚSTRIA. 1993, São Paulo. Anais. São Paulo: Signus, 1993, p.231-239.

PIRANI, Nicolas de Camargo. Sustentabilidade e a gestão compartilhada dos resíduos sólidos no município de Ribeirão preto/SP: conflitos e desafios. São Carlos,2010. Dissertação apresentada ao Programa de Pós-Graduação em ciências Sociais da Universidade Federal de São Carlos, 2010.

RIBEIRO, D.V.; MORELLI, M.R. Resíduos Sólidos: problema ou oportunidade? $1^{\mathrm{a}}$ Edição. Rio de Janeiro: Editora Interciência, 2009. 158 p.

SINHA-KHETRIWAL, Deepali; KRAEUCHI, Philipp; SCHWANINGER, Markus. A comparison of electronic waste recycling in Switzerland and in India. Environmental Impact Assessment Review, v. 25, n. 5, p. 492-504, 2005.

SIQUEIRA, Valdilene S.; MARQUES, Denise Helena França. GESTÃO E DESCARTE DE RESÍDUOS ELETRÔNICOS EM BELO HORIZONTE: ALGUMAS CONSIDERAÇÕES. Caminhos de Geografia, v. 13, n. 43, 2012.

TENÓRIO e ESPINOSA, J. A. S. e D. C. R. , Curso de Gestão Ambiental. Editora Manole, 2004. 